Ciência Florestal, Santa Maria, v. 24, n. 4, p. 899-910, out.-dez., 2014

ISSN 0103-9954

\title{
AVALIAÇÃO DE UM MÉTODO DE ANÁLISE SILVIGÊNICA EM UM CERRADÃO
}

\author{
EVALUATION OF A METHOD OF SILVIGENIC ANALYSIS IN CERRADÃO
}

\author{
Rejane Tavares Botrel $^{1}$ Kikyo Yamamoto ${ }^{2}$ Ricardo Ribeiro Rodrigues ${ }^{3}$
}

\begin{abstract}
RESUMO
O método "inventário de árvores de dossel por interceptação de linhas" foi aplicado para mapear o mosaico silvigênico em uma área de 5,12 ha de cerradão na Estação Ecológica de Assis (SP), e avaliado quanto à sua aplicabilidade para estimar o grau de conservação e para evidenciar os processos de regeneração neste tipo de floresta com dossel aberto. Também foi avaliada a distribuição de espécies tolerantes ou intolerantes à sombra entre as categorias de ecounidades. O mosaico compreendeu cinco clareiras (ecounidades em reorganização) e 1296 indivíduos, sendo 845 em ecounidades em equilíbrio, 241 em desenvolvimento, e 210 em degradação. Coerentemente com o dossel aberto induzindo reiteração precoce, predominaram as ecounidades em equilíbrio 2B (árvores altas com fuste baixo). Vochysia tucanorum Mart., Xylopia aromatica (Lam.) Mart. E Plenckia populnea Reissek, espécies intolerantes à sombra, ocorreram preferencialmente em ecounidades iniciais da silvigênese. Mas, a maioria das espécies, principalmente as tolerantes à sombra, não se associou com quaisquer categorias de ecounidades. $\mathrm{O}$ método evidenciou a maturidade e o bom estado de conservação arquitetural do cerradão estudado. O estudo mostrou que pode haver sobreposição de ecounidades em desenvolvimento e em degradação quando estas últimas são delineadas por árvores mortas em pé que permitem o crescimento de indivíduos jovens ao redor delas.

Palavras-chave: caracterização sucessional; ecounidades; mosaico florestal; silvigênese.
\end{abstract}

\begin{abstract}
The method "line-intercept sampling method of canopy trees" was applied to map the silvigenic mosaic of a patch of 5.12 ha in a cerradão at Estação Ecológica de Assis, in São Paulo state, aiming to verify its applicability in estimating the conservation status and to show the regeneration processes in this type of forest with open canopy. We also analyzed the distribution of shade tolerant or intolerant species among the categories of eco-unities. The mosaic comprised five tree fall gaps (eco-unities in reorganization) and 1,296 individuals, 845 of which in eco-unities in equilibrium, 241 in development, and 210 degrading. The predominance of eco-units in equilibrium 2B (tall trees with low pole) is coherent with the open canopy. The shade intolerant species Vochysia tucanorum, Xylopia aromatica and Plenckia populnea presented occurred preferably in starting eco-units of silvigenesis. But most of the species, especially the shade tolerant ones, did not present any correlation with eco-unit categories. The method revealed the maturity and the good architectural conservation status of the studied cerradão. We noticed that there may be overlapping of eco-unities in development and degrading when this latter one is delimited by dead standing trees that allow the growth of young individuals around them.
\end{abstract}

Keywords: eco-units; forest mosaic; silvi-genesis; sucessional characterization.

1 Engenheira Florestal, Dra ${ }^{\mathrm{a}}$., Professora Adjunto, Departamento de Ciências Vegetais, Universidade Federal Rural do Semiárido, Av. Francisco Mota, 572, Bairro Costa e Silva, CEP 59625-900, Mossoró (RN), Brasil. rtbotrel@ufersa.edu.br

2 Biologa, Dr ${ }^{\mathrm{a}}$.,Professora, Instituto de Biologia, Departamento de Botânica, Universidade Estadual de Campinas, Cidade Universitária Zeferino Vaz, Rua Monteiro Lobato, 255, Caixa Postal 6109, CEP 13083-862, Campinas (SP), Brasil. Kikyo@unicamp.br

3 Biologo, Dr., Professor, Departamento de Ciências Biológicas, Escola Superior de Agricultura Luiz de Queiroz, Av. Pádua Dias, 11, Caixa Postal 09, CEP 13418-900, Piracicaba (SP), Brasil. rrr@esalq.usp.br

Recebido para publicação em 8/02/2011 e aceito em 27/05/2013

Ci. Fl., v. 24, n. 4, out.-dez., 2014 


\section{INTRODUÇÃO}

O cerradão é uma fitofisionomia do bioma Cerrado que consiste numa formação florestal com dossel descontínuo e aspectos xeromórficos, e que se caracteriza pela presença de espécies de cerrado e também de espécies de floresta estacional semidecidual. Apesar da presença destas últimas, Rizzini (1979) observa que as condições ambientais no cerradão, principalmente o regime de luz, não permitem que a classificação ecológica de suas espécies em categorias sucessionais seja a mesma de uma mata mesófila.

Entretanto, visando estabelecer bases para a recuperação de áreas no Cerrado, Durigan et al. (2004) propuseram distinguir suas espécies entre as categorias de tolerantes e intolerantes à sombra, sendo esta classificação baseada em dados de literatura e em observações empíricas sobre os seus locais de distribuição no campo. $\mathrm{O}$ raciocínio implícito na proposta desta classificação seria análogo ao aplicado em análises de sucessão secundária em florestas que se iniciam em clareiras.

Alternativamente à análise da regeneração florestal através da dinâmica de clareiras, Oldeman (1978) propôs o estudo da dinâmica de florestas tropicais através da arquitetura arbórea, mais precisamente nas alterações arquiteturais ao longo do processo de construção da floresta que Hallé et al. (1978) definiram como silvigênese. E, seguindo a abordagem de Oldeman $(1978,1983)$, Torquebiau (1986) propôs um método de campo intitulado "interceptação de linhas para inventário das árvores do dossel" para mapear o mosaico florestal. Este método foi concebido e aplicado inicialmente em Florestas Úmidas de Dipterocarpáceas na Indonésia, com dossel contínuo, no interesse de avaliar, de modo simples e rápido, o estado geral da floresta para fins de exploração de madeira sem prejuízo de sua capacidade de autorregeneração. Assim, o autor pretendia obter informações da floresta como um todo, o tamanho, a quantidade e a distribuição de clareiras, isto é, de ecounidades em reorganização, das ecounidades em desenvolvimento que seriam formadas por árvores ainda juvenis, das ecounidades em equilíbrio que seriam compostas por árvores maduras, já reiteradas e com capacidade reprodutiva, e das ecounidades em degradação, compostas por árvores senis ou mortas.

A rapidez com que o método de Torquebiau (1986) pode ser executado para avaliar o grau de conservação e a capacidade de regeneração de um fragmento florestal como um todo, independente de sua utilidade para fins de exploração sustentável, é um grande atrativo. Mas ainda foi pouco testado no Brasil. Além do estudo de Engel e Prado (1992) em mata de tabuleiro no Espírito Santo, com dossel contínuo, constam poucos estudos no estado de São Paulo, como os de Cardoso-Leite (1995), Oliveira (1997), Botrel (2007) e Silva (2013) em florestas estacionais semideciduais, com dossel periodicamente mais aberto, Vanini (2009) em floresta de restinga e Lima et al. (2011) em floresta ombrófila densa. Em todos estes estudos, o método de Torquebiau (1986) produziu mosaicos florestais que evidenciaram o grau de conservação e o nível de perturbação das áreas de estudo, sugerindo que este poderia gerar o mesmo tipo de resultado em outros tipos florestais como, por exemplo, o cerradão, cujo dossel é sempre descontínuo.

Por outro lado, o estudo de Botrel (2007) é o único até o momento que analisou o método quanto à correspondência entre as categorias de ecounidades que representariam as fases do ciclo silvigênico e as fases correspondentes de sucessão secundária. E esta análise revelou a inexistência de uma correspondência clara e coerente, com fortes evidências de que o regime de luz decorrente da deciduidade foliar sazonal no tipo florestal estudado amplia as áreas de distribuição de espécies iniciais da sucessão secundária (pioneiras e secundárias iniciais) para setores maduros da floresta, onde o dossel mais fechado se torna permeável à luz nos períodos de perda foliar. Espera-se que resultado semelhante seja obtido no cerradão que, embora seja uma floresta perenifólia, possui dossel naturalmente aberto. E, se esse for o caso, a classificação de espécies tolerantes ou intolerantes à sombra com a expectativa de distinguir espécies iniciais e mais tardias no processo de sucessão secundária em cerradão não seria satisfeita.

Assim, ainda são desconhecidos os significados biológicos do inventário resultante da aplicação do método de Torquebiau (1986) e, portanto, qual ou quais podem ser os usos que se podem atribuir a este método para mapear o mosaico silvigênico, sobretudo em florestas com dossel descontínuo. Diferentemente do mosaico sucessional descrito por Whitmore (1975), com variações da composição florística e/ou dos parâmetros quantitativos dessas espécies na floresta, o mosaico silvigênico (sensu OLDEMAN, 1978) baseia-se na premissa de que a arquitetura das árvores de setores da floresta são testemunhas dos fenômenos 
de variação no regime de entrada de luz. Perguntase então se a análise silvigênica nestes moldes é ou não aplicável a florestas abertas como o cerradão. Além disso, o método de inventário e mapeamento do mosaico silvigênico propõe dispensar dados taxonômicos, mas o próprio autor do método (TORQUEBIAU, 1986) observa que a arquitetura das árvores pode depender de suas características genéticas, gerando diferentes respostas ao estímulo de luz. Até o momento, dados taxonômicos (florísticos) foram relacionados com as análises de natureza silvigênica apenas por Botrel (2007), e com resultados que geraram questionamentos tanto quanto ao método de inventário silvigênico como à classificação sucessional das espécies (grupos funcionais).

Assim, surgem questões como: estudos dinâmicos baseados na arquitetura das árvores da floresta são eficientes para diagnosticar sua fase silvigênica ou dos componentes do seu mosaico? $\mathrm{O}$ método de análise do mosaico silvigênico, concebido para florestas com dossel contínuo, pode gerar resultados em florestas com dossel aberto com significados biológicos equivalentes? $\mathrm{O}$ mosaico resultante da utilização de um método que se baseia unicamente na arquitetura arbórea dos indivíduos é ou não dependente das espécies que ocorrem na área de estudo? O mosaico silvigênico gerado pelo método de Torquebiau (1986) numa formação florestal com dossel aberto pode discriminar etapas silvigênicas que correspondem a fases sucessionais?

Norteados por estas questões mais gerais sobre o método, este foi avaliado no presente estudo em um trecho de cerradão, floresta de dossel aberto, na Estação Ecológica de Assis - SP, tendo por objetivos: (1) verificar se o método de interceptação de linhas para inventário de árvores do dossel proposto por Torquebiau (1986) permite evidenciar o estado de conservação de cerradão e distinguir ecounidades que representam as fases do ciclo silvigênico neste tipo de floresta; (2) testar a hipótese de que, devido ao dossel aberto e permeável à luz, as espécies classificadas como intolerantes à sombra não devem predominar em ecounidades de fases iniciais do ciclo silvigênico, e que espécies tolerantes à sombra não devem, necessariamente, predominar em ecounidades em equilíbrio, isto é, formadas por árvores maduras.

\section{MATERIAL E MÉTODOS}

\section{Área de estudo}

A Estação Ecológica de Assis, criada em 1992, localiza-se no oeste do Estado de São Paulo, sob as coordenadas $22^{\circ} 35^{\prime} \mathrm{S}$ e $50^{\circ} 22^{\prime} \mathrm{W}$, e constitui um dos últimos remanescentes da forma fisionômica cerradão, que originalmente recobria aquela região. Com uma área de 1312,38 ha, a Estação se encontra sobre a Formação Adamantina do grupo Bauru. O relevo regional é de colina, predominando declividades baixas de até $15 \%$ e amplitudes locais inferiores a $100 \mathrm{~m}$. Os dois tipos de solo predominantes são o Latossolo vermelho-escuro álico e o Podzólico vermelho-amarelo eutrófico.

O tipo climático, de acordo com a classificação de Köppen, é Cwa (mesotérmico) e a precipitação anual média é de $1480 \mathrm{~mm}$, com o período chuvoso se concentrando de outubro a março (DURIGAN et al., 1995). No interior dessa Unidade de Conservação, foi implantada uma parcela de $320 \times 320 \mathrm{~m}$, totalizando 10,24 ha, subdividida em 256 subparcelas contíguas de 20 x 20 m (400 $\mathrm{m}^{2}$ ). Essa área representa uma das quatro parcelas estabelecidas pelo Projeto Temático Diversidade, dinâmica e conservação em florestas do Estado de São Paulo: 40 ha de parcelas permanentes (Parcelas Permanentes) que integra o programa BIOTA/FAPESP. Para este trabalho, que integra o projeto Parcelas Permanentes, foi utilizada metade da parcela permanente, correspondendo a 5,12 ha.

\section{Caracterização silvigênica}

O método utilizado para o levantamento dos dados no campo foi o de interceptação de linhas para inventário das árvores do dossel (TORQUEBIAU, 1986). No interior da parcela estudada, foram dispostas linhas paralelas entre si e distantes $10 \mathrm{~m}$ umas da outra. As árvores dominantes, ou seja, aquelas com maior altura nos pontos de interceptação das linhas, tiveram seu número identificador, sua altura total $(\mathrm{Ht})$, altura do fuste (Hf), diâmetro a altura do peito (DAP) $\geq 4,8$ $\mathrm{cm}$, coordenadas (x e y) de localização e projeção horizontal anotados, utilizando como referência as subparcelas de $20 \times 20 \mathrm{~m}$. As áreas de clareira que interceptaram as linhas também foram amostradas, medidas e incluídas no mapeamento. $\mathrm{O}$ conceito de clareira utilizado nesta pesquisa foi o mesmo adotado por Engel (1993) e Cardoso-Leite (1995), 
ou seja, as aberturas no dossel que proporcionam uma superfície sem cobertura vegetal no solo de no mínimo $2 \mathrm{~m}$ de diâmetro.

Seguindoo métododetalhadoporTorquebiau (1986), as árvores incluídas no levantamento realizado entre 27/01/2004 a 24/07/2004 foram divididas quanto à sua arquitetura em: árvores do futuro (juvenis sem reiteração), árvores do presente (adultas com reiteração) e árvores do passado (com sinais de senescência ou mortas em pé). As árvores do presente foram subdivididas em duas categorias: árvores mais altas (categoria 1) ou mais baixas (categoria 2) que a superfície de inversão ecológica (PE) (metade da altura máxima do dossel). Em seguida, cada uma destas duas categorias foi dividida em duas subcategorias (A e B) considerando o ponto de inversão morfológica (PI). A razão altura do fuste (Hf)/altura total $(\mathrm{Ht})$, representa o PI $(\mathrm{PI}=\mathrm{Hf} / \mathrm{Ht})$, que ocorre quando a árvore diminui seu crescimento em altura (OLDEMAN, 1978). A posição de PI em relação ao PE separa as categorias A e B e sugere as condições de luminosidade, maior ou menor, nas quais o indivíduo reiterou. Se PI $>$ PE (subcategoria $\mathrm{A})$ e se $\mathrm{PI}<\mathrm{PE}$ (subcategoria $\mathrm{B}$ ). Assim, as árvores "A" possuem fuste longo e as árvores "B", fuste curto.

O mapeamento das ecounidades foi realizado manualmente em papel quadriculado (escala 1:10), usando as coordenadas das copas das árvores registradas nas linhas de inventário, originado o mosaico silvigênico. As clareiras constituem as ecounidades em reorganização, enquanto que a união das copas de árvores de mesma categoria delimita as ecounidades (a) em desenvolvimento, ocupadas por árvores do futuro; (b) em equilíbrio, ocupadas por árvores do presente e classificadas segundo os tipos de árvore que as compõem (1A, 1B, 2A ou 2B) e (c) em degradação, ocupadas por árvores do passado.

O mapa de ecounidades foi georreferenciado e finalizado por meio do programa TNT mips versão 6.8. O georreferenciamento do mapa de ecounidades foi realizado a partir das coordenadas fornecidas pela equipe do projeto Parcelas Permanentes. De posse dessa informação, os arquivos originais (extensão .JPEG) foram importados para o programa TNT mips (6.8) e o mapa de ecounidades foi colocado em suas coordenadas reais no sistema de referência South American Datum 1969 (SAD69).

\section{Levantamento florístico}

A listagem florística utilizada nesta pesquisa foi fornecida pela equipe técnica do Projeto Parcelas Permanentes. Além da composição de espécies, foram fornecidos dados de densidade e frequência dos indivíduos. Segundo o método descrito neste projeto, o levantamento florístico incluiu todos os indivíduos com DAP (diâmetro a altura do peito) igual ou superior a $4,8 \mathrm{~cm}$, ou PAP (perímetro a altura do peito) igual ou superior a $15,0 \mathrm{~cm}$. As amostras do material botânico-testemunho foram depositadas no Herbário ESA (ESALQ/USP). O levantamento florístico produziu um mapa das espécies com DAP superior a $4,8 \mathrm{~cm}$ que, posteriormente, foram classificadas em grupos ecológicos para serem confrontadas com o mapa de ecounidades.

\section{Classificação ecológica das espécies}

Adotou-se a proposta de Durigan et al. (2004) de se distinguir duas categorias ecológicas para as espécies ocorrentes em áreas de Cerrado: 1) Tolerante à sombra: espécies que podem se desenvolver e regenerar na sombra; e 2) Intolerante à sombra: espécies que só se desenvolvem e se regeneram em plena luz. Durigan et al. (2004) apresentam uma lista baseada em levantamentos florísticos realizados em fragmentos de vegetação nativa na Bacia do Médio Paranapanema (FRANCO, 2002, DURIGAN, 1999, DURIGAN; LEITÃO FILHO, 1995, MEIRA NETO, 1991, SILVEIRA, 2001) e dados não publicados do Banco de Dados do Projeto Viabilidade da Conservação dos Remanescentes de Cerrado no Estado de São Paulo (BIOTA - FAPESP) e Banco de Dados do Plano de Desenvolvimento Sustentável para o Entorno da Estação Ecológica de Assis (FNMA).

\section{Análise dos dados}

O mapa contendo as espécies arbóreas com $\mathrm{DAP} \geq 4,8 \mathrm{~cm}$ na área de estudo fornecido pelo Projeto Parcelas Permanentes e o mapa do mosaico silvigênico obtido neste estudo foram confrontados e analisados da seguinte forma: (1) Para verificar a relação entre espécies e categorias de ecounidades foi elaborada uma tabela (não apresentada) a partir do cruzamento do mapa do mosaico silvigênico com o censo, por meio do programa TNT mips (6.8). Essa tabela contém as espécies arbóreas e suas densidades absolutas nas diferentes ecounidades 
componentes do mosaico silvigênico. Espécies que ocorreram com menos que 10 indivíduos na área foram excluídas das análises, pois essa característica (frequência muito baixa) é inadequada para avaliar a distribuição espacial das mesmas dentro do mosaico silvigênico. Nesta tabela, o mapa do mosaico silvigênico (distribuição espacial de ecounidades) e as espécies arbóreas foram considerados como variáveis. Para analisar a associação entre estas variáveis, foi aplicado um teste de Qui-quadrado $\left(\mathrm{X}^{2}\right)(\mathrm{ZAR}, 1999)$ para testar a hipótese de que a frequência de ocorrência de uma variável (mosaico silvigênico) é dependente da frequência da segunda variável (espécies arbóreas), isto é, para verificar se as espécies arbóreas ocorrem preferencialmente em alguma categoria de ecounidade. Quando o teste de $\mathrm{X}^{2}$ apresentou resultado significativo, foi realizada uma análise de resíduos da tabela de contingência para analisar o comportamento de cada espécie arbórea presente na área de estudo (com 10 ou mais indivíduos) em relação às categorias de ecounidades. Tanto para verificar a associação entre as variáveis no teste de $\mathrm{X}^{2}$ quanto para as categorias de variáveis na análise de resíduos, seguiu-se Pereira (2001) e foi adotado o nível de significância de $1 \%$, para o qual o resíduo deve ser superior a $+2,58$ (excesso de ocorrências: associação positiva) ou inferior a $-2,58$ (falta de ocorrências: associação negativa). (2) Para analisar a distribuição espacial dos grupos ecológicos dentro do mosaico silvigênico. Foi realizado um novo cruzamento utilizando o programa TNT mips (6.8), entre o mapa do mosaico silvigênico e o do censo, sendo os indivíduos identificados segundo sua classificação ecológica de acordo com Durigan et al. (2004). Novamente foi gerada uma tabela de contingência para um novo teste de $\mathrm{X}^{2}$. Também foi realizada a análise de resíduos ajustados para verificar a existência de associação entre as categorias de ecounidades e os grupos ecológicos, da mesma forma explicitada anteriormente.

\section{RESULTADOS E DISCUSSÃO}

\section{Caracterização silvigênica}

A altura máxima encontrada para as árvores constituintes do mosaico silvigênico foi de $15 \mathrm{~m}$. No dossel superior da área estudada foram amostrados cinco clareiras (chablis) e 1296 indivíduos (253ind/ ha) distribuídos entre $845(65,20 \%)$ árvores do presente, 241 (18,59\%) árvores do futuro, 210 $(16,20 \%)$ árvores do passado. O mosaico silvigênico foi formado por muitas ecounidades pequenas. Houve predomínio de ecounidades em equilíbrio que totalizaram $74,12 \%$ da área mapeada (Tabela 1 ), seguidas de ecounidades em degradação com $14,79 \%$ e ecounidades em desenvolvimento com $10,63 \%$. As ecounidades em reorganização, representadas pelas clareiras, ocuparam apenas $0,47 \%$ do mapa. Dentre as ecounidades em equilíbrio, houve predomínio de ecounidades 2B $(43,46 \%)$. As árvores que constituem as ecounidades da categoria 2 se encontram entre as mais altas no levantamento, e as árvores $2 \mathrm{~B}$ se diferenciam daquelas que constituem as ecounidades $2 \mathrm{~A}(24,22 \%)$ por apresentarem o ponto de inversão morfológica (altura do fuste) abaixo do seu ponto de inversão ecológica (metade de sua altura total) (TORQUEBIAU, 1986). As ecounidades em equilíbrio 1A e 1B ocuparam,

TABELA 1: Estrutura das ecounidades no mosaico silvigênico em 5,12 ha de cerradão no interior da parcela permanente alocada na Estação Ecológica de Assis, Assis - SP.

TABLE 1: Eco-units structure of the mosaic silvigenic in 5.12 ha of cerradão within the permanent plot allocated at EstaçãoEcológica de Assis, Assis, SP state.

\begin{tabular}{cccccc}
\hline Ecounidade & Área mín $\left(\mathrm{m}^{2}\right)$ & Área máx $\left(\mathrm{m}^{2}\right)$ & Área total $\left(\mathrm{m}^{2}\right)$ & $\begin{array}{c}\text { Área média } \\
\left(\mathrm{m}^{2}\right)\end{array}$ & Área total (\%) \\
\hline Equilíbrio 1A & 8,33 & 93,35 & $1.821,01$ & 26,01 & 3,56 \\
Equilíbrio 1B & 10,36 & 152,32 & $1.469,38$ & 56,51 & 2,87 \\
Equilíbrio 2A & 11,82 & 989,22 & $12.385,57$ & 145,71 & 24,22 \\
Equilíbrio 2B & 18,46 & $4.193,61$ & $22.225,42$ & 358,47 & 43,46 \\
Reorganização & 22,59 & 128,42 & 238,50 & 47,70 & 0,47 \\
Desenvolvimento & 7,94 & 222,96 & $5.435,82$ & 53,29 & 10,63 \\
Degradação & 6,77 & 502,21 & $7.562,22$ & 87,93 & 14,79 \\
\hline
\end{tabular}


respectivamente, $3,56 \%$ e $2,87 \%$ da área.

A Tabela 2 mostra a contribuição percentual de ecounidades nos mosaicos silvigênicos, de diferentes áreas florestais, mapeadas pelo método de Torquebiau (1986). Apesar das diferenças, florestas ombrófilas (TORQUEBIAU, 1986, ENGEL; PRADO, 1992) e estacionais semideciduais (CARDOSO-LEITE, 1995; OLIVEIRA, 1997; BOTREL, 2007) apresentaram predomínio de ecounidades em equilíbrio $2 \mathrm{~A}$, as quais, de acordo com Torquebiau (1986), correspondem grosseiramente à clássica "fase madura" da floresta (WHITMORE, 1975), ou seja, zonas com árvores altas com longos fustes, usualmente com várias camadas intermediárias e um nível inferior sombreado, com poucas plantas pequenas. Ainda segundo Torquebiau (1986), as alturas variáveis dos níveis de inversão morfológica refletem as condições de crescimento dentro da floresta, as quais, por sua vez, influenciam a arquitetura arbórea: quanto mais baixo e mais aberto o dossel, menores seriam os fustes das árvores e quanto mais alto e mais fechado o dossel, maiores seriam os fustes das árvores. Mas estas são condições observáveis em florestas com dossel contínuo, pelo menos nos períodos em que não ocorre deciduidade foliar, e não em um cerradão onde o dossel é sempre aberto. De fato, no mosaico silvigênico do cerradão estudado, predominaram as ecounidades em equilíbrio $2 \mathrm{~B}$, o que é coerente com a maior permeabilidade à luz, o que induz a reiteração precoce das árvores.

Numa floresta estacional semidecidual em Piracicaba - SP, Oliveira (1997) relacionou as ecounidades em equilíbrio 2B com clareiras relativamente grandes. No entanto, a ocorrência de poucas clareiras e de tamanho relativamente pequeno não permite essa associação no cerradão estudado. Diferentemente, as árvores constituintes das ecounidades 2B do cerradão da E. E. de Assis parecem ter se desenvolvido sob uma matriz florestal baixa e/ou muito provavelmente sem um dossel contínuo, o que condiz com as características fisionômicas do cerradão e com a hipótese de que este pode ter evoluído a partir de um cerrado stricto sensu onde o nível de sombreamento é inferior ao do cerradão atual.

Ecounidades em equilíbrio compostas por árvores de menor porte (1A e 1B) podem resultar de distúrbios no dossel superior que geraram aberturas que permitiram o desenvolvimento de suas árvores constituintes (TORQUEBIAU, 1986). Árvores constituintes das ecounidades $1 \mathrm{~A}$ e $1 \mathrm{~B}$, de acordo com Torquebiau (1986), estariam anteriormente presentes no estrato médio da floresta, e teriam sido expostas à luz mediante distúrbios mais recentes (árvores 1A) ou menos recentes (árvores 1B). No entanto, dado o caráter descontínuo do dossel do cerradão, entende-se que fatores não relacionados ao regime de luz, por exemplo, os edáficos, poderiam produzir árvores com estas arquiteturas.

Comparado às florestas ombrófilas e as estacionais semideciduais, o cerradão estudado apresentou porcentagem muito baixa $(0,47 \%)$ de ecounidades em reorganização (Tabela 2). Este resultado pode ser devido a uma característica frequentemente observada nos cerradões, que é a

TABELA 2: Distribuição de área (\%) das ecounidades nos mosaicos silvigênicos mapeados através do método de inventário proposto por Torquebiau (1986), em diferentes tipos florestais.

TABLE 2: Distribution of area (\%) of the eco-units in silvigenic mosaics mapped through the inventory method proposed by Torquebiau (1986), in different forest types.

\begin{tabular}{ccccccc}
\hline Ecounidade & Cerradão $^{1}$ & FES $^{2}$ & FES $^{3}$ & FES $^{4}$ & Mata de Tabuleiros $^{5}$ & Florestas Úmida Dipt. $^{6}$ \\
\hline Equilíbrio 1A & 3,56 & 1,64 & 7,9 & 20,32 & 5,5 & 2,8 \\
Equilíbrio 1B & 2,87 & 2,00 & 2,9 & 6,66 & 11,7 & 0,3 \\
Equilíbrio 2A & 24,22 & 60,11 & 57,0 & 27,42 & 42,4 & 66,8 \\
Equilíbrio 2B & 43,46 & 12,98 & 16,0 & 18,8 & 23,4 & 11,4 \\
Reorganização & 0,47 & 6,17 & 5,3 & 10,9 & 3,2 & 4,8 \\
Desenvolvimento & 10,63 & 2,94 & 6,36 & 10,8 & 9,4 & 12,4 \\
Degradação & 14,79 & 14,16 & 3,46 & 5,1 & 4,3 & 1,4 \\
\hline
\end{tabular}

Em que: ${ }^{1}$ Cerradão (este estudo); ${ }^{2}$ Floresta Estacional Semidecidual (BOTREL, 2007); ${ }^{3}$ Média de três áreas em Floresta Estacional Semidecidual (CARDOSO-LEITE, 1995); ${ }^{4}$ Floresta Estacional Semidecidual (OLIVEIRA, 1997); ${ }^{5}$ Floresta Ombrófila Densa (ENGEL; PRADO, 1992); e ${ }^{6}$ Média de três áreas em Floresta úmida de Dipterocarpáceas (TORQUEBIAU, 1986). 
TABELA 3: Lista das espécies que apresentaram valores absolutos de resíduos ajustados (RA) superiores a 2,58 no cruzamento com as categorias de ecounidades. Grupos ecológicos (GE) das espécies: $\mathrm{T}=$ Tolerante à sombra; $\mathrm{I}=$ Intolerante à sombra.

TABLE 3: List of species that had absolute values of adjusted residuals (AR), greater than 2.58 at the junction with the categories of eco-units. Ecological group (GE) of the species: $\mathrm{T}=$ shade Tolerant, I = shade Intolerant

\begin{tabular}{ccccc}
\hline Espécie & \multirow{2}{*}{ GE } & Ecounidade \\
\cline { 3 - 5 } & & $-3,75$ & $-2,51$ & Equilíbrio \\
\hline Copaifera langsdorfii Desf. & T & $-1,23$ & 3,06 & $-1,27$ \\
Maytenus robusta Reissek & T & 4,30 & $-0,12$ & $-3,32$ \\
Plenckia populnea Reissek & I & 3,19 & 3,63 & $-5,18$ \\
Vochysia tucanorum Mart. & I & $-0,59$ & 5,26 & $-3,38$ \\
Xylopia aromatica (Lam.) Mart. & I & &
\end{tabular}

presença de árvores mortas em pé, possivelmente devido ao profundo sistema subterrâneo das árvores, o que dificulta a formação e o delineamento no mosaico de clareiras de grandes dimensões. Além disso, Rebertus e Burns (1997) notaram que a fisionomia savânica nos Estados Unidos pode ter origem em incêndios que desencadeiam processos de queda de árvores. A ausência de registros de incêndios recentes no cerradão da E. E. de Assis talvez explique, ao menos em parte, porque as clareiras na área de estudo são poucas e pequenas.

Compostas por clareiras (chablis) com luz abundante, ecounidades em reorganização em florestas com dossel contínuo representam a fase inicial da renovação da floresta. Entretanto, ao comparar com floresta estacional semidecidual, Muniz (2004) registrou variação relativamente menor na quantidade de luz entre as áreas mais e menos sombreadas no cerradão estudado. Ou seja, parecem não existir condições muito distintas de luminosidade que faça das pequenas e poucas clareiras os locais especiais para o início da silvigênese. Do ponto de vista funcional do mosaico florestal, Pélissier (1997) observa que podem existir dois tipos de silvigênese em função da dinâmica global de uma área florestal. O primeiro deles seria a partir da regeneração em chablis, seguindo o ciclo silvigênico proposto por Hallé et al. (1978). Mas este não parece ser o caso do cerradão. $\mathrm{O}$ segundo tipo seria por meio da morte em pé de indivíduos do dossel superior, que proporciona o crescimento de indivíduos jovens preexistentes, o que parece ser mais coerente com os dados encontrados no cerradão. Este segundo tipo de silvigênese também pode ser creditado à descontinuidade do dossel no cerradão, o qual proporciona entrada constante de luz com possibilidade de aumentos sazonais em todos os níveis da floresta.

As ecounidades em degradação (14,79\%), constituídas pelas árvores com claros sinais de senescência e muitas vezes mortas em pé, foram superiores em porcentagem quando comparadas àquelas encontradas em outros estudos de silvigênese (Tabela 2). Em tipos florestais com dossel mais fechado que o cerradão, ecounidades em degradação representam futuras clareiras (potenciais ecounidades em reorganização). Entretanto, como a maioria das árvores do passado do cerradão permanecem em pé depois de mortas, indivíduos suprimidos do sub-bosque podem desenvolverse em árvores do futuro. Se as árvores do passado forem mais altas que as juvenis em desenvolvimento abaixo delas, é a ecounidade em degradação que será amostrada neste local pelo método de Torquebiau (1986). Quando há sobreposição de ecounidades em desenvolvimento e em degradação e, as ecounidades em desenvolvimento tendem a ficar subamostradas e pode-se ter a impressão de uma passagem rápida ou direta de ecounidade em degradação para ecounidade em equilíbrio.

\section{Mosaico silvigênico $\times$ fatores bióticos (florísticos)}

$\mathrm{O}$ resultado do teste de $\mathrm{X}^{2}$ realizado para verificar a existência de associação entre as espécies ocorrentes na área de estudo e as categorias de ecounidades, foi significativo no nível de $1 \%(219,02)$ indicando que as variáveis não são independentes. De acordo com a análise de resíduos ajustados (RA) (Tabela 3), somente cinco espécies, que serão descritas a seguir, contribuíram para o resultado do teste de $\mathrm{X}^{2}$. Ou seja, apenas 
estas apresentaram associação com as categorias de ecounidades do mosaico.

Dentre as cinco espécies que apresentaram associação positiva ou negativa com alguma categoria de ecounidade, há duas tolerantes à sombra (Copaifera langsdorfii e Maytenus robusta), e três intolerantes à sombra (Vochysia tucanorum, Xylopia aromatica e Plenckia populnea). O padrão espacial de indivíduos com DAP $\geq 4,8 \mathrm{~cm}$ de três destas espécies, Vochysia tucanorum, Xylopia aromatica e Copaifera langsdorfii foi estudado por Capretz (2004) na parcela permanente de cerradão da $E$. E. de Assis. O autor encontrou diferentes níveis de agregação para estas três espécies, evidenciando suas preferências por determinados locais, tal como mostram estes resultados. Isto é, estas espécies de fato possuem distribuição espacial não aleatória.

Copaifera langsdorfii ( $\mathrm{T})$, espécie representada pelo maior número de indivíduos na área de estudo, apresentou associação negativa com ecounidades em degradação e positiva com as ecounidades em equilíbrio (Tabela 3 ). Sendo tolerante à sombra é aceitável que ela se associe positivamente com as ecounidades em equilíbrio, formadas por indivíduos de copa mais ampla que produzem sombreamento sobre elas. Mas Maytenus robusta (T) apresentou associação contrária à de Copaifera langsdorfii, isto é, positiva com ecounidades em desenvolvimento que são formadas por árvores com arquitetura não reiterada e que podem permitir maior entrada de luz no sub-bosque. Este resultado pode ser explicado pelo estudo de Berkenbrock e Paulilo (1999), onde foram observadas respostas positivas das sementes de Maytenus robusta tanto às condições de luz como de escuro durante o processo germinativo. Além disso, estes autores detectaram maior crescimento inicial das plântulas sob maior intensidade de luz, indicando que esta espécie pode ser intolerante à sombra pelo menos em sua fase jovem. Mas Maytenus robusta é citada ainda como típica de submata por Gomes et al. (2005), num estudo realizado em floresta ombrófila densa montana em Pindamonhangaba - SP, o que sugere que esta pode ser uma espécie ecologicamente plástica. A situação desta espécie remete à dificuldade de se enquadrar as espécies arbóreas em grupos ecológicos estáveis, visto que, além da plasticidade ecológica, as características de tolerância à luz podem variar ao longo da ontogenia.

Vochysia tucanorum (I) apresentou associação positiva com ecounidades em degradação e em desenvolvimento, e associação negativa com as ecounidades em equilíbrio. Este resultado é coerente com os estudos de Van den Berg e Oliveira Filho (1999), assim como de Barbosa et al. (1999), que registram a ocorrência frequente desta espécie em ecótonos, variando de formações vegetais densas a mais abertas, mas com preferência por locais mais iluminados como borda de clareira ou de matas. Barbosa et al. (1999) também mostraram que a germinação e o crescimento inicial desta espécie podem ocorrer sob diversas condições de luz. Ou seja, esta é mais uma espécie que apresenta plasticidade ecológica. A ocorrência preferencial desta espécie em ecounidades em degradação e em desenvolvimento no cerradão estudado pode indicar a presença de clareiras antigas nessas áreas, embora esta não seja uma exigência, pois a espécie consegue germinar e se desenvolver também em locais sombreados. Conforme já analisado anteriormente, muitas ecounidades em degradação foram delineadas na área por indivíduos mortos em pé. E estes, segundo Oldeman (1978), proporcionam gradualmente as mesmas mudanças energéticas que ocorrem nas clareiras propriamente ditas, e proporcione o tipo de silvigênese descrito por Pélissier (1997), onde ecounidades em degradação e em desenvolvimento podem sobrepor-se no tempo e no espaço. E isso explicaria a associação positiva da heliófita Vochysia tucanorum com ecounidades em degradação.

Xylopia aromatica (I) associou-se positivamente com ecounidades em desenvolvimento e negativamente com ecounidades em equilíbrio; e Plenckia populnea (I) associou-se positivamente com ecounidades em degradação, e negativamente também com ecounidades em equilíbrio. O resultado de Xylopia aromatica pode ser explicado de modo análogo ao de Vochysia tucanorum, Xylopia aromatica, citado por Almeida et al. (1998), como sendo mais abundante em áreas mais abertas, sendo encontrada frequentemente em áreas de Cerrado com alta luminosidade. A associação negativa dessa espécie com as ecounidades em equilíbrio reforça essa observação. Já os dados aqui dispostos não permitem explicar ecologicamente porque Plenckia populnea associou-se positivamente com ecounidades em degradação e negativamente com ecounidades em equilíbrio. Mas, do ponto de vista da dinâmica populacional, este resultado pode estar relacionado com a incapacidade ou a dificuldade local desta espécie em se reproduzir ou de recrutar novos indivíduos. 
Mosaico silvigênico $\times$ fatores bióticos (ecológicos)

$\mathrm{O}$ resultado do teste de $\mathrm{X}^{2}$ para verificar associações entre a classificação ecológica aplicada aos indivíduos e as categorias de ecounidades foi significativo no nível de $1 \%(11794,12)$, indicando a dependência entre as variáveis. A análise de resíduos ajustados (Tabela 4) indicou somente associações positivas entre o grupo intolerante à sombra e as categorias de ecounidades em reorganização e em desenvolvimento. Ecounidades em reorganização são formadas por clareiras e compreendem locais mais iluminados. Ecounidades em desenvolvimento também são presumidamente melhor iluminadas que as ecounidades em equilíbrio. Então, é coerente que indivíduos intolerantes à sombra ocorram preferencialmente nestes ambientes. Dale (2000) observa que as variações espaciais da luz podem levar os indivíduos de uma mesma espécie a serem encontrados juntos em áreas com condições favoráveis, resultando num padrão agregado. No Cerrado em Itirapina - SP, Miranda-Melo (2004) encontrou resultados indicativos de que o padrão espacial de indivíduos adultos da espécie intolerante à sombra Xylopia aromatica seria parcialmente determinado pela luz, o que é compatível com estes resultados (Tabela 3 ).

Porém, chama a atenção o fato de que indivíduos classificados como tolerantes à sombra não se associaram a nenhuma categoria de ecounidade, indicando serem indiferentes ao ambiente que ocupam em relação à luz dentro do mosaico. Como a maioria das espécies inventariadas é classificada como tolerante à sombra, e este grupo ecológico não apresentou associação com nenhuma das categorias de ecounidades, isto significa que a maioria das espécies do cerradão apresentou distribuição espacial aleatória em relação às fases do ciclo silvigênico na área de estudo. Há um estudo que Lieberman et al. (1995) realizaram em 11,11 ha de floresta tropical de terras baixas não perturbadas em La Selva, Costa Rica, onde estimaram um índice de fechamento de dossel acima das árvores com DAP $\geq 10 \mathrm{~cm}$. Neste estudo, $86,5 \%$ das 90 espécies encontradas estavam distribuídas aleatoriamente em relação ao fechamento do dossel, ocupando indiscriminadamente as condições de disponibilidade de luz. Este resultado é compatível com as presentes observações em cerradão, embora muito mais notável devido ao maior fechamento de dossel na floresta que os autores estudaram. Assim, é possível supor que tanto em florestas com dossel contínuo como em florestas com dossel aberto, podem existir algumas poucas espécies que expressam mais fortemente as suas preferências ou necessidades quanto à luz ou à sombra, enquanto que a maioria das espécies pode ser relativamente plástica e capaz de explorar condições variadas de sombreamento ou iluminação.

\section{CONCLUSÕES}

O método de interceptação de linhas para inventário de árvores do dossel proposto por Torquebiau (1986) evidenciou o bom estado de conservação arquitetural do cerradão estudado, assim como o seu estado de maturidade, com predomínio de ecounidades em equilíbrio, principalmente do tipo $2 \mathrm{~B}$, o que é coerente com o tipo florestal com dossel aberto. Pela arquitetura arbórea, o método distinguiu as categorias de ecounidades e revelou ainda que existem poucas e pequenas clareiras (ecounidades em reorganização) na área, o que pode estar relacionado à tendência das árvores no cerradão de permaneceram mortas em pé. Esta característica pode gerar uma sobreposição, no tempo e no espaço, de fases do ciclo silvigênico. Ou seja, é possível que, à exceção

TABELA 4: Valores dos resíduos ajustados (RA - valores absolutos superiores a 2,58 em negrito) resultantes do cruzamento das categorias de ecounidades com os grupos ecológicos.

TABLE 4: Values of adjusted residuals (RA - absolute values greater than 2.58 in bold type) that resulted from the crossing of the eco-unit categories and the ecologic groups.

\begin{tabular}{|c|c|c|c|c|c|}
\hline \multirow{2}{*}{ Ecounidade } & \multicolumn{2}{|c|}{ Classificação Ecológica } & \multirow{2}{*}{ Ecounidade } & \multicolumn{2}{|c|}{ Classificação Ecológica } \\
\hline & I & $\mathrm{T}$ & & I & $\mathrm{T}$ \\
\hline Equilíbrio 1A & 1,66 & $-0,66$ & Reorganização & 2,87 & $-1,15$ \\
\hline Equilíbrio 1B & 1,11 & $-0,44$ & Desenvolvimento & 3,16 & $-1,26$ \\
\hline Equilíbrio $2 \mathrm{~A}$ & $-1,49$ & 0,59 & Degradação & 0,70 & $-0,28$ \\
\hline Equilíbrio 2B & $-2,19$ & 0,88 & & & \\
\hline
\end{tabular}


das ecounidades em equilíbrio, que contêm árvores maduras, as demais categorias de ecounidade não obrigatoriamente representam fases silvigênicas 'puras'. Isto porque as ecounidades em degradação, delineadas muitas vezes por árvores mortas em pé, são tão permeáveis à luz que podem equivaler a clareiras. Além disso, elas podem funcionar como sítios de desenvolvimento das árvores jovens que se encontravam abaixo da copa das árvores antes delas morrerem. Assim, a silvigênese no cerradão não ocorreria segundo o modelo de regeneração a partir de uma clareira típica como nas florestas com dossel contínuo.

Devido ao dossel aberto e permeável à luz, esperava-se que as espécies classificadas como intolerantes à sombra não predominariam em ecounidades de fases iniciais do ciclo silvigênico. Mas foram exatamente algumas espécies intolerantes à sombra que predominaram em ecounidades que representam estas fases iniciais da silvigênese. Mas confirma-se parcialmente a expectativa de que as espécies tolerantes à sombra não necessariamente predominariam em ecounidades em equilíbrio. De fato, as espécies classificadas como tolerantes à sombra, que foram a maioria na área de estudo, apresentaram distribuição aleatória entre categorias de ecounidades. Recoleta de dados a intervalos de tempo regulares na área de estudo poderão ajudar a acompanhar a evolução do ciclo silvigênico e a aprofundar a compreensão do significado biológico dos mosaicos delineados pelo método de interceptação de linhas e inventário para árvores do dossel em cerradão.

\section{REFERÊNCIAS BIBLIOGRÁFICAS}

ALMEIDA, S. P. de, et al. Cerrado: espécies vegetais úteis. Planaltina: EMBRAPA-CPAC, 1998. $464 \mathrm{p}$.

BARBOSA, A. R., YAMAMOTO, K; VALIO, I. F. M. Effect of light and temperature on germination and early growth of VochysiatucanorumMart., Vochysiaceae, in cerrado and forest soil under different radiation levels. Revista Brasileira de Botânica, São Paulo v. 22, n. 2 (suplemento), p. 275-280, 1999.

BATALHA, M. A.;MARTINS, F. R. Floristic, Frequence, and Vegetation Life-Form Spectra of a Cerrado Site, Brazilian Journal of Biology, v. 64, n. 2, p. 203-209, 2004.

BERKENBROCK, I. S.;PAULILO, M. T. S.. Efeito da luz na germinação e no crescimento inicial de
Maytenus robusta Reiss. eHedyosmum brasiliense Mart. Revista Brasileira de Sementes, Brasília, v. 21, n. 2, p. 243-248, 1999.

BOTREL, R. T.. Análise silvigênica em floresta estacional semidecidual e em cerradão no estado de São Paulo. 2007. 106 f. Tese (Doutorado em Biologia Vegetal) - Universidade Estadual de Campinas, Campinas, 2007.

BOURLIERE, F.; HADLEY, M. The Ecology of Tropical Savannas, Annual Review of Ecology and Systematics, v. 1, p. 125-152, 1970.

CARDOSO-LEITE, E. Ecologia de um fragmento florestal em São Roque, SP: florística, fitossociologia e silvigênese. 1995.118f. Dissertação (Mestrado em Biologia Vegetal) - Universidade Estadual de Campinas, Campinas, 1995.

CAPRETZ, R. L. Análise dos padrões espaciais de árvores em quatro formações florestais do estado de São Paulo, através de análises de segunda ordem, como a função $k$ de Ripley. 2004. 40 f. Dissertação (Mestrado em Ecologia de Agroecossistemas) - Escola Superior de Agricultura Luiz de Queiroz, Piracicaba, 2004.

COSTA, A. A.; ARAÚJO, G. M. de. Comparação da vegetação arbórea de cerradão e de cerrado na Reserva do Panga, Uberlândia, Minas Gerais. Acta Botanica Brasilica, São Paulo, v. 15, n. 1, p. 63-72, 2001.

COSTA, I. R. da; ARAÚJO, F. S. de.; LIMAVERDE, L. W. Flora e aspectos auto-ecológicos de um encrave de cerrado na Chapada do Araripe, Nordeste do Brasil. Acta Botânica Brasilica, São Paulo, v. 18, n. 4, p. 759-770, 2004.

COUTINHO, L. M.. O conceito de Cerrado. Revista Brasileira de Botânica, São Paulo, v. 1, p. 17-23, 1978.

COUTINHO, L. M. Fire in the ecology of the Brazilian Cerrado. In: GOLDAMMER, J. G., ed.. Fire in the tropical biota: ecosystem processes and global challenges. Berlim: Springer-Verlag. 1990.

DALE, M. R. T. Spatial pattern analysis in plant ecology. Cambridge: University Press. 2000. 326 p. DURIGAN, G. et al. Plano de manejo da Estação Ecológica de Assis, Assis, São Paulo. 1995.

DURIGAN, G.;LEITÃO FILHO, H. F. Florística e fitossociologia de matas ciliares do oeste paulista. Revista do Instituto Florestal, v. 7, p. 197-239, 1995.

DURIGAN, G.; GURGEL-GARRIDO, L. M. A.; GARRIDO, M. A. O.. Desenvolvimento de Plathymeni areticulata Benth em plantio puro e em consorciação com espécies de diferentes estádios 
sucessionais. Revista do Instituto Florestal, São Paulo, v. 11, p. 131-136, 1999.

DURIGAN, G.; SILVEIRA, E. R.. Recomposição da mata ciliar em domínio de cerrado, Assis, SP, ScientiaForestalis, v. 56, p. 135-144, 1999.

DURIGAN, G. et al. Caracterização de dois estratos da vegetação em uma área de Cerrado, Acta Botanica Brasílica, São Paulo, v. 16, p. 251-262, 2002.

DURIGAN, G. et al. A flora arbustivo-arbórea de Médio Paranapanema: base para a restauração dos ecossistemas naturais. In: VILLAS BOAS, O.; DURIGAN, G., orgs. Pesquisas em conservação e recuperação ambiental no Oeste paulista: resultados da cooperação Brasil/Japão. 2004

ENGEL, V. L.; PRADO, P. I. K. L. Aspectos da silvigênese de uma Mata Pluvial Atlântica em Linhares, ES In: CONGRESSO NACIONAL SOBRE ESSÊNCIAS NATIVAS, 2., 1992, São Paulo. Anais.... São Paulo: Instituto Florestal, 1992. v. 4, p. 163-168.

ENGEL, V. L. Silvigênese, dinâmica de fragmentos e a conservação de florestas tropicais. Série técnica florestal, Botucatu v.1, p.1-21, 1993.

FELFILI, J. M.; ASSUNÇÃO, S. L. Fitossociologia de um fragmento de cerrado sensu stricto na APA do Paranoá, DF, Brasil, Acta Botanica Brasílica, São Paulo, v. 18, p. 903-909, 2004.

FIDELIS, A. T.; GODOY, S. A. P. de. Estrutura de um cerrado stricto sensu na gleba cerrado péde-gigante, Santa Rita do Passa Quatro, SP, Acta Botanica Brasílica, São Paulo, v. 17, p. 531-539, 2003.

FRANCO, G. A. D. C. Florística e fitossociologia de duas unidades do mosaico florestal da Estação Ecológica dos Caetetus - Floresta Estacional Semidecidual, Gália - SP. 2002. 47 f. Dissertação (Mestrado em Ciências) - Escola Superior de Agricultura Luiz de Queiroz, Piracicaba, 2002.

FURLEY, P. A; RATTER, J. A. Soil resources and plant communities of the central braziliancerrado and their development. Journal of Biogeography, v. 15 , p. $97-108,1988$.

GOMES, B. Z., MARTINS, F. R. ; TAMASHIRO, J. Y. Estrutura do cerradão e da transição entre cerradão e floresta paludícola num fragmento da InternationalPaper do Brasil Ltda., em Brotas, SP. Revista Brasileira de Botânica, v. 27, p. 249-262, 2004.

GOMES, E. P. C.; FISCH, S. T. V.; MANTOVANI, W. Structureoftreecomponent in Reserva Ecológica do Trabijú, Pindamonhangaba, São Paulo State,
Brazil. Acta Botanica Brasílica, São Paulo, v. 19, p. 451-464, 2005.

HALLÉ ,F., OLDEMAN, R. A. A.; TOMLINSON, P. B. Tropical Trees and forests: an architectural analysis. Berlim: Springer-Verlag. 1978.

LIEBERMAN, M. et al. Canopy closure and the distribution of tropical forest tree species at $\mathrm{La}$ Selva, Costa Rica. Journal of Tropical Ecology, v. 11, p. 161-178, 1995.

MEIRA NETO, J. A. A. Composição florística e fitossociológica da vegetação de Cerrado Sensu Lato da Estação Ecológica de Santa Bárbara (E.E.S.B.), Município de Águas de Santa Bárbara. Estado de São Paulo. 1991. 49 f. Dissertação (Mestrado em Biologia) - Universidade de Campinas, Campinas, 1991.

MIRANDA-MELO, A. A. Estrutura de população de Xylopia aromática (Lam.) Mart. (Annonaceae) e RoupalamontanaAubl. (Proteaceae) em quatro fragmentos de cerrado sensu lato no município de Itirapina/SP. 2004. 52 f. Dissertação (Mestrado em Biologia Vegetal) Instituto de Biologia, Universidade Estadual de Campinas, Campinas, 2004.

MUNIZ, M.R.A. Estudo do regime de luz nas quatro principais formações fitogeográficas no estado de São Paulo durante o inverno do ano de 2003. 2004. 95 f. Dissertação (Mestrado em Biologia Vegetal) -Universidade Estadual de Campinas, Campinas, 2004.

OLDEMAN, R. A. A. Architeture an energy exchange of dicotyledonous trees in the forest. In: TOMLINSON, P. B.; ZIMMERMANN, M. H., eds Tropical trees as living systems. Cambridge: University Press, 1978. p. 535-560.

OLDEMAN, R. A. A. Tropical rainforest, architeture, sylvigenesis and diversity. In: SUTTON, S. L.; WHITMORE, T. C.; CHADWICK, A. C., eds. Tropical rainforest: ecology and management. Oxford: Blackwell Scientific, p. 139-150. 1983.

OLIVEIRA, R.E.. Aspectos da dinâmica de um fragmento florestal em Piracicaba-SP: silvigênese e ciclagem de nutrientes. 1997. 40 f. Dissertação (Mestrado em Ciências Florestais) - Escola Superior de Agricultura Luiz de Queiroz, Piracicaba, 1997.

PELISSIER, R. Hétérogénéité spatiale et dynamique d'une forêt dense humide dans les Ghats occidentaux de l'Inde. Institut français de Pondichéry, Inde, Publications du département d'écologie v. 37, p. 1-148. 1997.

PROGRAMA DE GEOREFERENCIAMENTO TNTmips.versão 6.8 for Windows. 
PEREIRA, J. C. R. Análise de dados qualitativos: estratégias metodológicas para asciências da saúde, humanas e sociais. 3. ed. São Paulo: EDUSP, 2001. $157 \mathrm{p}$.

RATTER, J. A. et al. Observations on the vegetation of northeastern MatoGrosso: 1. The woody vegetation types of the XavantinaCachimbo expedition area. Philosophical Transactions ransactions of the Royal Society of London. 1973.

RATTER, J. A.; RIBEIRO, J. F.; BRIDGEWATER, $\mathrm{S}$. The braziliancerrado vegetation and threats to its biodiversity. Annals of Botany, v. 80, p. 223-230, 1997.

REBERTUS, A. J.; BURNS, B. R.. The importance of gap processes in the development and maintenance of oak savannas and dry forests.Journal of Ecology, v. 85, p. 635-645, 1997.

RIBEIRO, J. F.; WALTER, B. M. T. Fitofisionomias do Bioma Cerrado. In: SANO, S.; ALMEIDA, S. P., Eds. Cerrado: ambiente e flora. Planaltina: EMBRAPA-CPAC. 1998. p. 89-166.

RIZZINI, C.T. Tratado de fitogeografia do Brasil
- aspectos sociológicos e florísticos. São Paulo: HUCITEC/ Ed. Universidade de São Paulo,. 1979. $374 \mathrm{p}$.

SILVEIRA, E. R. Recuperação da mata ciliar do córrego Tarumã (Tarumã, SP): aspectos estruturais, florísticos e ambientais de quatro diferentes modelos florestais, dez anos após o plantio. 2001. 84 f. Dissertação (Mestrado em Ciências da Engenharia Ambiental) - Escola de Engenharia de São Carlos, Universidade de São Paulo, São Carlos, 2001.

TORQUEBIAU, E.F. Mosaic patterns in dipteriocarp rainforest in Indonesia and their implications for pratical forestry. Journal of Tropical Ecology, v. 2, p. 301-325, 1986.

VAN DEN BERG, E.; OLIVEIRA FILHO, A.T. Spatial partitioning among tree species within an area of tropical montane gallery forest in southeastern Brazil. Flora, v. 194, p. 249-266, $1999 .$.

WHITMORE, T.C. Tropical rain forest of the ear east . Oxford: Claredon Press, 1975.

ZAR, J. H. 1999. Bioestatistical analysis. New Jersey, Prentice Hall. 663 p. 